\title{
Factores predictores de la actividad física en escolares españoles de acuerdo a su estado de peso Predictive factors of physical activity in Spanish students based on their weight status
}

\author{
Alejandro Martínez-Baena, Daniel Mayorga-Vega, Jesús Viciana
}

Universidad de Valencia (España), **Universidad de Granada (España)

Resumen: El objetivo del presente estudio fue comparar la motivación autodeterminada hacia el ejercicio físico, autoconcepto físico, barreras hacia la actividad física e intención de ser físicamente activo entre adolescentes con y sin sobrepeso/obesidad. Una muestra de 333 escolares, de 13 a 16 años, participaron en esta investigación. Para la medición de las distintas variables se utilizaron instrumentos validados: Índice de masa corporal, Behavioral Regulation in Exercise Questionnaire, Physical Self Description Questionnaire, Autoinforme de Barreras para la Práctica de Ejercicio Físico e Intention to Be Physically Active Questionnaire. Los resultados mostraron diferencias estadísticamente significativas de acuerdo al índice de masa corporal (sobrepeso/obesidad) en todas las variables analizadas tanto en chicos como en chicas $(p<0,05)$, excepto en la intención de ser físicamente activo. La comprensión de los factores predictores o determinantes de la actividad física analizados, resulta relevante por ser estos potencialmente modificables. Por tanto, adaptar programas de intervención orientados a promocionar actividad física a las evidencias encontradas ayudaría a aumentar los índices de participación activa en escolares, especialmente en aquellos con sobrepeso u obesidad.

Palabras clave: Factores predictores, actividad física, adolescentes, masa corporal, escuela.

Abstract: The aim of the present study was to compare self-determined motivation towards exercise, physical self-concept, barriers towards physical activity, and intention to be physically active among adolescents with and without overweight/obesity. A sample of 333 schoolchildren aged 13 to 16 years old participated in this research study. Validated instruments were used for the measurement of the variables included in the study: Body Mass Index, Behavioral Regulation in Exercise Questionnaire, Physical Self Description Questionnaire, Self-report of Barriers towards the Practice of Exercise, and Intention to Be Physically Active Questionnaire. The results showed statistically significant differences by body mass index (overweight/ obesity) in all analyzed variables in both boys and girls $(p<0.05)$, except for the intention to be physically active. Understanding the analyzed predictive factors or determinants of physical activity is essential because they are potentially modifiable. Therefore, using the evidences found in our study to adapt physical activity promotion intervention programs would help increasing the rates of active participation in schoolchildren, especially those who are overweight or obese.

Keywords: Predictive factors, physical activity, adolescents, body mass, school.

\section{Introducción}

Diversos estudios han centrado su interés en analizar los factores predictores de la participación en la actividad física (AF) por parte de los adolescentes (Sallis, Prochaska \& Taylor, 2000; Biddle, Gorely \& Stensel, 2004) con la finalidad de que asuman hábitos de vida más activos querepercutan en una mejora de su salud(Beltrán, Sierra, Jiménez, González-Cutre, Martínez \& Cervello, 2017). Algunos estudios de revisión (Van der Horst, Paw, Twisk \& Van Mechelen, 2007; Park \& Kim, 2008; Dolenc \& Pisot, 2010; Sterdt, Liersch \& Walter, 2014), resumen estos factores. De acuerdo a dicha literatura, los mismos podrían agruparse en factores demográficos y biológicos, psicológicos, conductuales, sociales, ambientales, las propias características de la AF u otros, como los nuevos estilos de vida juveniles. El estudio de los mismos, resulta esencial debido a los bajos niveles de participación y cumplimiento de recomendaciones de práctica deAF(Oviedo, Sánchez, Castro, Calvo, Sevilla, Iglesias \& Guerra, 2013; Pulido, Sánchez-Oliva, Sánchez-Miguel, González-Ponce \& García-Calvo, 2016), demostrándose altos índices de sedentarismo entre dicho grupo de población (Noriega et al., 2015). Por tanto, conocerlos y analizarlos en profundidad, ayudaría a orientar mejor la promoción de la AF para la consecución deniveles más elevados de participación activa (Bush \& MacDonald, 2015) tanto en el tiempo libre (Villa-González, Ruiz \& Chillón, 2016), como en el ámbito escolar por parte de los escolares (Abarca-Sos, Murillo, Julián, Zaragoza \& Generelo, 2015; Viciana, Martínez-Baena \& Mayorga-Vega, 2016), habiéndose demostrado una asociación positiva entre dichos factores y los niveles de AF de los mismos (Wang, Baranowski, Lau, Chen \& Zhang, 2016). LaAF de moderada a vigorosa de los adolescentes está influenciada por muchos factores, por lo que la eficacia de las intervenciones para promoverla sería mayor si estos determinantes fueran mejor comprendidos (Graham, Wall, Larson \& Neumark-Sztainer, 2014).

Respecto a los factores predictores señalados, el presente estudio centra su interés en el análisis de las variables interpersonales y psicoló-

Fecha recepción: 10-05-16. Fecha de aceptación: 27-04-17

Alejandro Martínez Baena

alejandro.martinez@uv.es gicas del escolar adolescente (Motivación autodeterminada hacia el ejercicio físico, Autoconcepto físico, Barreras hacia la AF e Intención de ser físicamente activo), encuadradas dentro de los modelos teóricos puramente psicosociales (Modelo de creencias sobre la salud, teoría de la motivación por protección, teoría de la acción razonada/teoría del comportamiento planeado, modelo de la AF, modelo transteórico). Estos factores han sido elegidos debido a las evidencias encontradas sobre los altos grados de correlación existentes entre dichos determinantes o factores predictores y el nivel de AF de los adolescentes (Sallis et al., 2000; Van der Horst et al., 2007; Park y Kim, 2008; Sterdt et al., 2014).

Precisamente Sterdt et al. (2014), señalan el conocimiento de estos factores psicológicos, cognitivos y emocionales como muy relevante por su notable incidencia en la AF de los adolescentes. Aún así, señalan que dicho conocimiento respecto a los factores predictores de la $\mathrm{AF}$ de niños y adolescentes sigue resultando insuficiente. En la misma línea, Marques, Peralta, Martins, Sarmento, Routen y Carreiro (2016) evocan la necesidad de seguir profundizando en la evidencia sobre los factores influyentes de tipo psicosocial asociados a la AF de dicho grupo de población. Atkin, Van Sluijs, Dollman, Taylor y Stanley (2016), se muestran más críticos y puntualizan que no se trataría de un problema de volumen de literatura orientada a describir los factores predictores o determinantes de la AF en población joven, sino basado en la inconsistencia de los hallazgos y mala calidad metodológica de los estudios publicados en este sentido.

Respecto al primer factor, diversos estudios han centrado su interés en analizar los procesos motivacionales asociados a la práctica de AF. La motivación es una característica psicológica que despierta el organismo y que provoca ciertos comportamientos dirigidos a una determinada meta. Es decir, se puede entender como la causa psicológica de cualquier acción. En este sentido, se demuestra como una variable que correlaciona positivamente, y de acuerdo con las diversas regulaciones conductuales, con el posterior comportamiento hacia la AF (Owen, Smith, Lubans, Ng \& Lonsdale, 2014). La teoría motivacional más utilizada para dicho propósito en los últimos años ha sido la Teoría de la Autodeterminación (TAD) (Deci \& Ryan, 1985). Dicha teoría presenta diversas formas motivacionales a lo largo de un continuum de autodeterminación, donde los extremos serían la motivación intrínseca y la desmotivación, situándose entre ellos otros tipos de motivación 
con mayor o menor grado de la misma (regulación externa, regulación introyectada, regulación identificada y regulación integrada). Respecto a ello, números estudios evidencian que formas de motivación más autodeterminadas se asocian con mayor adherencia al ejercicio, así como con mejores niveles deAF en población adolescente(Mayorga \& Viciana, 2014) y desde dicha teoría (Downs, Savage \& DiNallo, 2013).

Estudios orientados a analizar la motivación hacia el ejercicio físico en adolescentes de acuerdo a su peso, sólo encontramos el estudio de Gourlan, Trouilloud y Sarrazin (2013), cuyo objetivo se centró en conocer las características de la motivación de adolescentes obesos hacia la AF a partir de la Teoría de la autodeterminación y a través del uso de la versión francesa del Behavioral Regulation Exercise Questionnaire (BREQ-2), demostrándose que los adolescentes obesos no se involucran necesariamente en estilos de vida activos por razones intrínsecas tales como el placer o la diversión, sino más bien por los beneficios percibidos de la AF en términos de bienestar y salud. El estudio de Power, UllrichFrench, Steele, Daratha y Bindler (2011) centrado en examinar la relación entre la motivación y el peso en adolescentes con y sin sobrepeso, con la capacidad cardiorrespiratoria como factor mediador, evidenciando que aquellos adolescentes no obesos muestran una mayor motivación intrínseca hacia laAF que aquellos que si lo son, lo que repercutiría en contar con mayores niveles de actividad y condición física, quedando condicionado el IMC al grado de autodeterminación de la motivación. Y por último, el estudio de Hwang y Kim (2013) centrado en analizar la asociación entre el Índice de Masa Corporal (IMC) y la Teoría de la Autodeterminación como factores predictores de laAF en adolescentes con normopeso, sobrepeso y obesidad, a través de la versión coreana del BREQ-2, manifestándose que a pesar de que el grado de motivación intrínseca era mayor en adolescentes con normopeso que en aquellos con sobrepeso y obesidad, esto no se traducía en los niveles de AF moderada y sólo ligeramente en los de AF intensa.

El autoconcepto físico ha sido definido como las percepciones que tienen los sujetos sobre sus habilidades físicas y su apariencia física, constituyendo además una de las dimensiones que contribuyen a la formación del autoconcepto general (Marsh, Richards, Johnson, Roche \& Tremayne, 1994). La percepción de estas características adquiere especial relevancia en la adolescencia por ser un período de constitución de la personalidad, así como un elemento facilitador de relaciones sociales entre los escolares. En este sentido, se ha demostrado una asociación positiva entre los niveles de AF de los adolescentes y su autoconcepto físico (Liu, Wu \& Ming, 2015). Diversos estudios han examinado la relación de la actividad y condición física(CF) y la composición y masa corporal con el autoconcepto general y físico (Velez, Golem \& Arent, 2010; Zook, Saksvig, Kuo \& Young, 2012), utilizando el IMC como indicador de composición corporal. Fernández-Bustos, González-Martí, Contreras y Cuevas (2015), plantean que, si la insatisfacción influye en la percepción física y el IMC se relaciona con dicha insatisfacción, también debería relacionarse con el autoconcepto físico del adolescente. En este sentido, se ha constatado que un IMC alto se asociaría a un autoconcepto bajo en adolescentes (O’Dea, 2006). Reigal-Garrido, Becerra-Fernández, Hernández-Mendo y Martín-Tamayo (2014) demuestran una fuerte asociación entre el porcentaje de masa grasa y el autoconcepto en adolescentes. Destaca el estudio de García-Sánchez, Burgueño-Menjibar, López-Blanco y Ortega (2013), quienes determinaron la relación entre la cantidad de grasa corporal y el autoconcepto en adolescentes, al querer demostrar que a pesar de tener sobrepeso u obesidad se podrían tener buenos niveles de CF y un buen autoconcepto en los mismos.

Respecto al análisis de las barreras que dificultan la práctica de AF, Niñerola, Capdevilla y Pintanel (2006), recuerdan la necesidad de su abordaje de manera previa a la implementación de estrategias orientadas a aumentar la motivación y por tanto, la adherencia hacia el inicio y el mantenimiento de una conducta activa. De acuerdo a esto, estudios centrados en el análisis de la percepción de barreras respecto a la AF en adolescentes se vienen realizando desde hace años (Gunnell, Brunet, Wing \& Belanger, 2015). Sólo encontramos cuatro estudios que hayan analizado las posibles diferencias respecto a la percepción de barreras hacia la AF de acuerdo al peso en niños. De acuerdo al estudio de Zabinski, Saelens, Stein, Hayden-Wade y Wilfley (2003), los niños con sobrepeso podrían concebirse como más vulnerables respecto a la percepción de barreras relacionadas con el propio cuerpo, tales como ser conscientes del mismo o su propia apariencia cuando hacen AF o sentirse observados mientras la realizan. En la misma línea, Reesor, Maeda, Raedeke, McMillan y DuBose, (2014) evidenciaron que los niños con sobrepeso u obesidad podían percibir mayor número de barreras hacia la AF que los niños sanos, pero que esa mayor percepción de las mismas no afectaba en los niveles de AF de moderada a vigorosa. Respecto a estudios con adolescentes, destacamos el de Deforche, De Bourdeaudhuij y Tanghe (2006), quienes constataron una asociación positiva entre la participación en AF y la actitud medida mediante la percepción de barreras hacia la práctica, comprobando que dicha asociación no era moderada por el nivel de sobrepeso. En cambio, en el estudio de Young-Ho (2008) se constataron diferencias significativas respecto a la percepción de barreras en adolescentes con y sin sobrepeso, constituyéndose las mismas como un factor predictor de AF en estos grupos de población y sugiriendo que las estrategias para mejorar la AF de los adolescentes con sobrepeso y obesidad deberían centrarse en la reducción de barreras relacionadas con su estado de peso.

Respecto al cuarto factor y según la teoría del comportamiento planificado, la actitud influye en la intención cuando uno es capaz de controlar el comportamiento (Ajzen, 1985). La intención es considerada como un factor predictor del comportamiento y se supone que refleja la fuerza o intensidad por hacer o participar en algo. Se intuye por tanto que la intención mostrada por los escolares respecto a la practica de AF podría ser un buen indicador de su motivación. En este sentido, Hein, Muur y Koka, (2004) ya demostraron que la motivación intrínseca por experimentar una práctica estimulante se constituye como el mayor predictor de intención de ser físicamente activo tras la etapa de escolarización obligatoria. Más allá de la motivación intrínseca por el logro, diversos estudios han centrado su interés en intentar predecir la intención de ser físicamente activo en adolescentes (Murtagh, Rowe, McMinn \& Nelson, 2012), mediante el uso de diversos modelos y teorías (Roberts, Maddison, Magnusson \& Prapavessis, 2010; Raudsepp, Viira \& Hannus, 2010; Park, 2011). Moreno, Moreno y Cervelló (2007), revelaron la relación entre el autoconcepto físico y la intención de los escolares de seguir siendo físicamente activos tras la etapa educativa, suponiendo esta investigación una primera aproximación al análisis de la intencionalidad de ser físicamente activo y del autoconcepto físico de forma conjunta. Más recientemente, Grao-Cruces, Fernández-Martínez, Nuviala y Pérez-Turpin (2015), analizaban la influencia de los niveles de AF, CF, sedentarismo y satisfacción con la vida, con dicha intención, demostrando que aquellos adolescentes que presentaban niveles bajos en estos parámetros, contaban también con una mayor probabilidad de presentar menor intención de ser físicamente activos al finalizar la educación secundaria. En cambio, no hemos encontrado estudios que determinen dicha variable de acuerdo al estado de peso en adolescentes.

Sterdt et al. (2014) recuerda que el conocimiento respecto a los factores predictores de la AF de niños y adolescentes sigue resultando insuficiente. Por tanto, una de las finalidades del estudio se centra en profundizar en el análisis de este tipo de factores psicológicos en población adolescente española. Además, no hemos encontrado ningún estudio centrado en analizar posibles asociaciones entre factores psicológicos predictores de AF en escolares de acuerdo a su estado de peso. Consecuentemente, el objetivo del presente estudio fue comparar la motivación autodeterminada hacia el ejercicio físico, autoconcepto físico, barreras hacia la AF e intención de ser físicamente activo entre adolescentes con y sin sobrepeso/obesidad.

Los adolescentes con sobrepeso-obesidad deberían mostrar una menor motivación hacia el ejercicio físico, autoconcepto físico e intención de ser físicamente activos, así como una mayor percepción de barreras hacia la AF. 


\section{Método}

\section{Participantes}

Un total de 394 adolescentes (211 varones y 183 mujeres), de 13 a 16 años de edad, fueron invitados a participar en el presente estudio. Los adolescentes eran estudiantes de $1^{\circ}-4^{\circ}$ curso de un centro concertado de educación secundaria situado en la ciudad de Granada (España). Todas las familias de los estudiantes tenían un estatus socioeconómico medio. De los adolescentes que fueron invitados, 349 aceptaron participar en el presente estudio. Sin embargo, debido a que 16 adolescentes con bajo masa corporal fueron excluidos, en el presente estudio 333 participantes fueron finalmente analizados.

Los criterios de inclusión fueron: a) estar matriculado en los cursos de $1^{\circ}-4^{\circ}$ de educación secundaria del centro seleccionado; b) no padecer ninguna enfermedad o lesión que les impidiera realizar AF con normalidad; c) presentar el consentimiento informado firmado por sus padres o tutores legales, y d) presentar el asentimiento informado por parte de los adolescentes. Los criterios de exclusión fueron: a) no participar en todas las sesiones de evaluación, y/o b) no tener un registro válido de ninguna de las variables de estudio.

\section{Medidas}

Composición corporal. La composición corporal se evaluó mediante el IMC (Ruiz et al., 2011). Primero se midió la masa corporal (Seca, Ltd., Hamburgo, Alemania; precisión = 0,1 kg) y la talla (Holtain Ltd., Crymmych, Pembs, Reino Unido; precisión = 0,1cm) y luego se calculó como la masa corporal dividida por la estatura al cuadrado (kg/ $\mathrm{m}^{2}$ ). Las medidas se realizaron con los participantes en camiseta y pantalón corto y descalzos. Se realizaron dos mediciones de cada variabley luego se calculó el promedio de cada una de ellas. El estado del peso de los participantes se categorizó posteriormente de acuerdo con los puntos de corte internacionales para el IMC como no-sobrepeso (IMC $<$ punto de corte de sobrepeso) y sobrepeso (IMC > punto de corte de sobrepeso) (Cole, Bellizzi, Flegal \& Dietz, 2000).

Motivación autodeterminada hacia el ejercicio físico. Se midió mediante la versión española del Behavioral Regulation in Exercise Questionnaire (BREQ-3), adaptada y validada por González-Cutre et al.(2010), la cual incluye la dimensión de la regulación integrada(Wilson, Rodgers, Loitz, \& Scime, 2006). El cuestionario consta de 23 ítems distribuidos en seis dimensiones que miden la motivación intrínseca, motivación integrada, tres formas de regulación para la motivación extrínseca (identificada, introyectada y externa) y la desmotivación. Cada dimensión es constituida por cuatro ítems (salvo la regulación identificada que constaba de 3 valorables en una escala tipo Likert de 5 puntos con las etiquetas de $1=$ «Nada verdadero» hasta $5=$ «Totalmente verdadero». El cuestionario está encabezado por la frase «Yo hago ejercicio físico...», y los ítems representan las diferentes razones de dicho enunciado, reflejando los diversos tipos de motivación. El índice RAI también se calcula a partir de las dimensiones de este cuestionario de la siguiente manera: 3 x motivación intrínseca +2 x regulación integrada + regulación identificada - regulación introyectada - 2 x regulación externa-3x desmotivación(Vallerand \& Rousseau, 2001). La versión española utilizada del BREQ-3 posee unas propiedades psicométricas adecuadas (CFI =0,91; RMSEA=0,06; Alfa de Cronbach =0,66-0,87) (González-Cutre, Sicilia \& Fernández, 2010).

Autoconcepto físico. Se midió mediante la versión corta y adaptada del Physical Self Description Questionnaire (PSDQ) de Marsh et al. (1994). El cuestionario consta de 40 ítems que miden nueve dimensiones específicas del autoconcepto físico (fuerza, AF, grasa corporal, coordinación, resistencia, competencia deportiva, salud, apariencia física y flexibilidad) y dos dimensiones globales (autoconcepto físico general y autoestima). Cada dimensión es constituida al menos por tres ítems valorables en una escala tipo Likert de 6 puntos con las etiquetas de $1=$ «Totalmente falso» hasta 6 = «Totalmente verdadero». La versión española utilizada del PSDQ-S posee unas propiedades psicométricas adecuadas ( $\mathrm{CFI}=0,98$; RMSEA = 0,05; Alfa de Cronbach $=0,70-0,88$ ) (Marsh, Martin \& Jackson, 2010).
Barreras hacia laAF. Se utilizó el Autoinforme de Barreras para la Práctica de Ejercicio Físico (ABPEF) adaptado y validado por Niñerola et al. (2006) a partir de la escala original de Capdevilla (2005). El cuestionario consta de 17 ítems distribuidos en cuatro dimensiones que miden Imagen corporal/Ansiedad física y social, Fatiga/Pereza, Obligaciones/Falta de Tiempo y Ambiente/Instalaciones. La primera dimensión está constituida por cinco ítems, la segunda por seis y la tercera y cuarta por tres, valorables en una escala tipo Likert de 11 puntos con las etiquetas de $0=$ «Poca» hasta $10=$ «Mucha». El cuestionario está encabezado por la frase «¿cuál es la probabilidad de que las siguientes razones te impidan realizar ejercicio físico?», y los ítems representan las diferentes razones que impiden practicar ejercicio en las próximas semanas, reflejando los diferentes tipos de barreras percibidas. La versión utilizada posee unas propiedades psicométricas adecuadas (Varianza explicada $=62,7 \%$; Alfa de Cronbach $=0,68-0,88$ ) (Niñerola et al., 2006).

Intención de ser físicamente activo. La intención de ser físicamente activo fuera de la escuela se midió mediante la versión española del Intention to Be Physically Active Questionnaire (Hein el al., 2004), adaptado y validado por Moreno et al. (2007). El cuestionario consta de 5 ítems: (1) «Me interesa el desarrollo de mi forma física», (2) «Al margen de las clases de Educación Física, me gusta practicar deporte», (3) «Después de terminar el instituto, quisiera formar parte de un club deportivo de entrenamiento», (4) «Después de terminar el instituto, me gustaría mantenerme físicamente activo/a», y (5) «Habitualmente practico deporte en mi tiempo libre». Los participantes respondieron a los ítems en una escala tipo Likert de 5 puntos con las etiquetas de 1 = «Totalmente en desacuerdo» hasta 5 = «Totalmente de acuerdo». La versión española del cuestionario ha demostrado unas propiedades psicométricas adecuadas (CFI=0,98; RMSEA = 0,06; Alfa de Cronbach $=0,94$ ) (Moreno et al., 2007).

\section{Procedimiento}

El estudio fue primero aprobado por el Comité de Ética de la Universidad de [eliminado por cuestiones de anonimato]. A continuación, un investigador contactó con el director y profesores de educación física del centro. Se les informó sobre el proyecto y se les solicitó permiso para llevar a cabo el estudio. Después de obtener la aprobación del centro, los estudiantes de $1^{\circ}-4^{\circ}$ curso y sus tutores legales fueron plenamente informados sobre la naturaleza y propósito del estudio. Se les informó que para que un alumno pudiera formar parte del estudio debía rellenar el asentimiento informado y su tutor legal el consentimiento informado. Asimismo, todo alumno tenía el derecho de no otorgar su consentimiento a participar o a revocarlo en cualquier momento del estudio sin que supusiera perjuicio alguno o discriminación. Por otro lado, se les informó que los datos personales recogidos en esta investigación serían anónimos y en todo momento se protegería la confidencialidad de los datos personales según la ley vigente.

La recogida de datos se realizó durante los meses de marzo-junio de 2014. Los cuestionarios fueron aplicados dentro del horario escolar (aproximadamente 15-20 minutos por cuestionario durante dos clases de EF), solicitándose seriedad y máxima sinceridad en las respuestas. La masa corporal y la estatura se midieron en otra clase de EF, de acuerdo a los protocolos estandarizados, pautas de seguridad e instrucciones establecidas en la batería utilizada.

\section{Análisis estadístico}

Se realizó una estadística descriptiva de la edad, masa corporal, talla, IMC y los resultados de las variables dependientes. Posteriormente, debido a que la mayoría de los datos no siguieron una distribución normal, se utilizó la prueba de U de Mann-Whitney para muestras independientes para estudiar las posibles diferencias entre adolescentes con normopeso y sobrepeso/ obesidad. Previamente los participantes se clasificaron en función de su IMC en: bajo peso, normopeso, sobrepeso y obesidad (Cole et al., 2000; Cole et al., 2007). Debido al bajo número de adolescentes con bajo peso (10 varones y 6 mujeres), dichos participantes no se seleccionaron. En el caso del sobrepeso y la 
obesidad, a causa del bajo número de adolescentes en algunas de las categorías (varones: 49 con sobrepeso y 18 con obesidad; mujeres: 27 con sobrepeso y 8 con obesidad) los datos fueron unidos. El tamaño de la muestra varía ligeramente para las distintas variables porque varios participantes no completaron algunos de los cuestionarios. Sin embargo, para hacer el máximo uso de los datos, todos los datos válidos se incluyeron en este estudio. Todos los análisis anteriores se realizaron separadamente para los varones y las mujeres. Estos análisis estadísticos se realizaron mediante el paquete estadístico SPSS versión 21.0 para Windows (IBM ${ }^{\circledR}$ SPSS ${ }^{\circledR}$ Statistics 21). El nivel de significación estadística se estableció en $p>0,05$.

Tabla

\begin{tabular}{lcccc}
\multicolumn{2}{c}{ Características generales (media \pm desviación estándar) de los participantes estudiados } \\
\cline { 2 - 5 } & \multicolumn{2}{c}{ Varones } & \multicolumn{2}{c}{ Mujeres } \\
\cline { 2 - 5 } & Normopeso & Sobrepeso/obesida & Normopeso & Sobrepeso/obesida \\
& $(n=116)$ & $\mathrm{d}(n=67)$ & $(n=115)$ & $\mathrm{d}(n=35)$ \\
\hline Edad (años) & $14,46(1,09)$ & $14,45(1,08)$ & $14,42(1,12)$ & $14,49(1,27)$ \\
Masa corporal $(\mathrm{kg})$ & $55,11(9,01)$ & $71,55(12,76)^{*}$ & $50,82(6,67)$ & $67,06(9,16)^{*}$ \\
Talla $(\mathrm{m})$ & $1,66(0,10)$ & $1,66(0,09)$ & $1,59(0,06)$ & $1,60(0,05)$ \\
IMC $\left(\mathrm{kg} / \mathrm{m}^{2}\right)$ & $19,87(1,79)$ & $25,82(3,05)^{*}$ & $20,05(1,97)$ & $26,00(2,49)^{*}$ \\
\hline Nota IMC = Índice de masa corporal. ${ }^{*} p<0,001$. & \multicolumn{3}{c}{}
\end{tabular}

Tabla 2 .

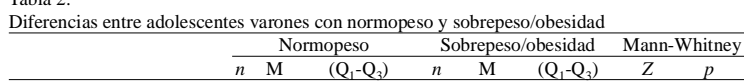

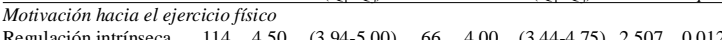

$\begin{array}{lllllllll}\text { Regulación intrínseca } & 114 & 4,50 & (3,94-5,00) & 66 & 4,00 & (3,44-4,75) & 2,507 & 0,012\end{array}$

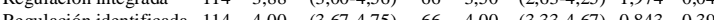

Regulación id

$\begin{array}{lllllllll}\text { Regulación } & 114 & 2,00 & (1,46-2,75) & 66 & 2,42 & (1,50-3,00) & 1,730 & 0,084\end{array}$

$\begin{array}{lllllllll}\text { introyectada } & 114 & 1,25 & (1,00-1,75) & 66 & 1,50 & (1,00-2,31) & 2,841 & 0,005\end{array}$

$\begin{array}{lllllllll}\text { Desmotivación } & 114 & 1,00 & (1,00-1,31) & 66 & 1,00 & (1,00-1,81) & 1,408 & 0,159\end{array}$

$\begin{array}{lllllll}\text { Motivación } & 114 & 15,17 & (12,25-19,98) & 66 & 13,63 & (6,52-17,54) \\ 2,672 & 0,008\end{array}$

autodeterminada

Autoconcepto físico

Salud

Coordinación

Actividad física

$\begin{array}{llllllll}112 & 4,23 & (3,80-5,20) & 66 & 4,00 & (3,48-4,80) & 2,106 & 0,035 \\ 112 & 4,75 & (4,00-5,50) & 66 & 4,38 & (3,69-5,25) & 1,335 & 0,182\end{array}$

$\begin{array}{lllllllll}\text { Competencia deportiva } & 112 & 4,83 & (4,00-5,67) & 66 & 4,33 & (3,33-5,33) & 2,591 & 0,010\end{array}$

$\begin{array}{lllllllll}\begin{array}{l}\text { Autoconcepto físico } \\ \text { global }\end{array} 112 & 5,00 & (4,08-5,67) & 66 & 4,00 & (3,00-5,00) & 4,846<0,001\end{array}$

$\begin{array}{lllllllll}\text { Apariencia física } & 112 & 4,33 & (3,33-5,00) & 66 & 4,00 & (2,67-4,75) & 1,137 & 0,255\end{array}$

$\begin{array}{lllllllll}\text { Fuerza } & 112 & 4,67 & (3,67-5,00) & 66 & 4,67 & (3,67-5,00) & 0,099 & 0,922\end{array}$

$\begin{array}{lllllllll}\text { Flexibilidad } & 112 & 3,33 & (2,67-4,00) & 66 & 3,33 & (2,00-4,00) & 0,700 & 0,484\end{array}$

$\begin{array}{llllllll}\text { Resistencia } & 112 & 4,67 & (4,00-5,33) & 66 & 4,00 & (2,67-4,67) & 3,922<0,001\end{array}$

$\begin{array}{lllllllll}\text { Autoestima } & 112 & 4,80 & (4,40-5,40) & 66 & 4,80 & (4,20-5,20) & 1,083 & 0,279\end{array}$ Barreras hacia la actividad física

Imagen

corporal/ansiedad física $114 \quad 0,60 \quad(0,00-2,20) \quad 66 \quad 1,00 \quad(0,00-3,60) \quad 1,817 \quad 0,069$

social

$\begin{array}{lllllllll}\text { Fatiga/ pereza } & 114 & 1,58 & (0,50-2,83) & 66 & 2,00 & (0,67-3,33) & 1,039 & 0,299\end{array}$

$\begin{array}{lllllllll}\text { Obligaciones/ Falta de } & 114 & 3,00 & (1,00-4,75) & 66 & 3,50 & (1,00-5,75) & 1,212 & 0,226\end{array}$

\begin{tabular}{llllllllll} 
tiempo & 114 & 3,00 & $(1,00-4,75)$ & 66 & 3,50 & $(1,00-5,75)$ & 1,212 & 0,226 \\
Ambiente/ instalaciones & 114 & 0,83 & $(0,00-2,00)$ & 66 & 0,67 & $(0,00-2,33)$ & 0,420 & 0,674 \\
\hline
\end{tabular}

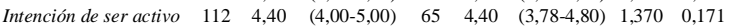
Nota. $\mathrm{M}=$ Mediana; $\mathrm{Q}_{1}=$ Primer cuartil; $\mathrm{Q}_{3}=$ Tercer cuartil.

Tabla 3.

Diferencias entre adolescentes mujeres con normopeso y sobrepeso/obesidad

$\begin{array}{ccc}\text { Normopeso } & \text { Sobrepeso/obesidad Mann-Whitney }\end{array}$

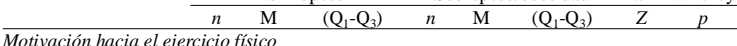
$\begin{array}{lllllllll}\text { Regulación intrínseca } & 110 & 3,88 & (2,75-4,50) & 35 & 4,00 & (3,00-4,75) & 0,839 & 0,402\end{array}$ $\begin{array}{lllllllll}\text { Regulación integrada } & 110 & 3,25 & (2,25-4,00) & 35 & 3,25 & (2,25-4,25) & 0,584 & 0,559\end{array}$ $\begin{array}{lllllllll}\text { Regulación identificada } & 110 & 4,00 & (3,00-4,38) & 35 & 4,00 & (3,33-4,33) & 0,091 & 0,928\end{array}$ $\begin{array}{lllllllll}\begin{array}{l}\text { Regulación } \\ \text { introyectada }\end{array} & 110 & 1,75 & (1,25-2,25) & 35 & 2,25 & (1,75-2,75) & 3,004 & 0,003\end{array}$ $\begin{array}{lllllllll}\text { Regulación externa } & 110 & 1,25 & (1,00-1,75) & 35 & 1,50 & (1,00-2,25) & 1,997 & 0,046\end{array}$ Desmotivación $\quad \begin{array}{llllllll}110 & 1,00 & (1,00-1,50) & 35 & 1,00 & (1,00-1,75) & 0,502 & 0,616\end{array}$

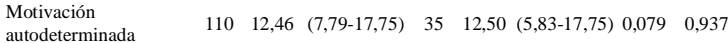
autodeterminada

$\begin{array}{lllllllll}\begin{array}{l}\text { Autoconcepto fisico } \\ \text { Salud }\end{array} & 111 & 5,40 & (4,80-5,80) & 35 & 5,40 & (4,60-5,80) & 0,695 & 0,487\end{array}$

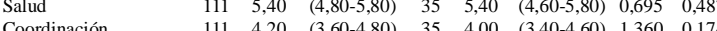
$\begin{array}{lllllllll}\text { Coordinación } & 111 & 4,20 & (3,60-4,80) & 35 & 4,00 & (3,40-4,60) & 1,360 & 0,174\end{array}$ $\begin{array}{lllllllll}\text { Actividad fisica } & 111 & 4,00 & (3,00-5,00) & 35 & 4,25 & (3,25-5,38) & 1,192 & 0,233\end{array}$ Competencia deportiva $111,4,00 \quad(3,00-4,4$ Competencia deportiva 111 $\begin{array}{lllllllll}\text { Autoconcepto físico } & 111 & 4,33 & (3,33-5,33) & 35 & 3,00 & (2,33-5,00) & 3,686<0,001 \\ \text { global }\end{array}$

$\begin{array}{lllllllll}\text { Apariencia física } & 111 & 3,67 & (3,00-4,67) & 35 & 3,67 & (2,67-4,33) & 0,978 & 0,328\end{array}$ $\begin{array}{lllllllll}\text { Fuerza } & 111 & 3,33 & (2,67-4,33) & 35 & 3,67 & (3,33-4,33) & 2,137 & 0,033\end{array}$ $\begin{array}{lllllllll}\text { Flexibilidad } & 111 & 4,00 & (3,00-5,00) & 35 & 3,67 & (2,67-5,00) & 0,772 & 0,440\end{array}$

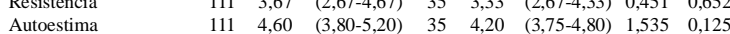
Barreras hacia la actividad fisica

Imagen

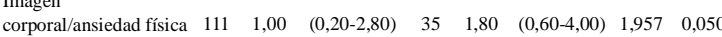

$\begin{array}{llllllllll}\text { Fatiga/ pereza } & 111 & 2,17 & (0,83-4,33) & 35 & 2,17 & (0,83-4,00) & 0,057 & 0,954\end{array}$ Obligaciones/ Falta de $\quad 111 \quad 4,00 \quad(2,00-5,67) \quad 35 \quad 3,33 \quad(1,67-6,00) \quad 1,223 \quad 0,22$ tiempo $\begin{array}{lllllllll}\text { Ambiente/ instalaciones } & 111 & 1,00 & (0,00-2,00) & 35 & 0,33 & (0,00-1,67) & 1,125 & 0,261\end{array}$ \begin{tabular}{lllllllll} 
Intención de ser activo & 108 & 3,80 & $(3,20-4,40)$ & 35 & 4,00 & $(3,40-4,60)$ & 1,479 & 0,139 \\
\hline$N$
\end{tabular} Nota. $\mathrm{M}=$ Mediana; $\mathrm{Q}_{1}=$ Primer cuartil; $\mathrm{Q}_{3}=$ Tercer cuartil.

\section{Resultados}

La Tabla 1 muestra las características de los participantes estudiados, así como las diferencias entre los adolescentes con normopeso y sobrepeso/obesidad. Tanto en varones como mujeres, los resultados de la prueba U de Mann-Whitney mostraron cómo los/as adolescentes con sobrepeso/obesidad presentaban mayores valores estadísticamente significativos de masa corporal e IMC que sus compañeros/as con normopeso $(p<0,001)$. En cambio, para la edad y la talla no se encontraron diferencias estadísticamente significativas $(p>0,05)$.

Las Tablas 2-3 muestran las diferencias en la motivación hacia el ejercicio físico, autoconcepto físico, barreras hacia la AF e intención de ser físicamente activo entre los adolescentes con normopeso y sobrepeso/ obesidad para los varones y mujeres, respectivamente.

Motivación autodeterminada hacia el ejercicio físico. Los resultados de la prueba U de Mann-Whitney mostraron cómo los adolescentes varones con normopeso reportaron mayores valores estadísticamente significativos de regulación intrínseca, regulación integrada eíndice de motivación autodeterminada que sus compañeros con sobrepeso/ obe$\operatorname{sidad}(p<0,05)$. En cambio, los varones con sobrepeso/obesidad reportaron mayores valores estadísticamente significativos de regulación externa que sus compañeros con normopeso $(p<0,01)$. En cuanto a las mujeres, los resultados mostraron cómo las adolescentes con sobrepeso/ obesidad reportaron mayores valores estadísticamente significativos en la regulación introyectada y externa que sus compañeras con normopeso $(p<0,05)$. Para el resto de dimensiones de la motivación hacia el ejercicio físico no se encontraron diferencias estadísticamente significativas $(p>0,05)$.

Autoconcepto físico. Los resultados de la prueba U de MannWhitney mostraron cómo los adolescentes varones con normopeso presentaban una autopercepción estadísticamente significativa mejor en la coordinación, grasa corporal, competencia deportiva, autoconcepto físico global y resistencia que sus compañeros con sobrepeso/obesidad $(p<0,05)$. En cuanto a las mujeres, los resultados mostraron cómo las adolescentes con normopeso presentaban una autopercepción estadísticamente significativa mejor en la grasa corporal y autoconcepto físico global que sus compañeras con sobrepeso/ obesidad $(p<0,001)$. En cambio, las adolescentes mujeres con sobrepeso/ obesidad reportaron una mejor autopercepción de la fuerza que sus compañeras con normopeso $(p<0,05)$. Para el resto de dimensiones del autoconcepto físico no se encontraron diferencias estadísticamente significativas ( $p>$ $0,05)$.

Barreras hacia la AF. Los resultados de la prueba U de MannWhitney mostraron cómo las adolescentes mujeres con sobrepeso/ obesidad percibieron estadísticamente una mayor barrera por imagen corporal/ansiedad física social que sus compañeras con normopeso ( $p$ $>0,05$ ). Demodo similar, hubo una tendencia a la significación en la que los adolescentes varones con sobrepeso/obesidad también percibieron mayores valores de barreras por imagen corporal/ansiedad física social que sus compañeros con normopeso $(p<0,10)$. En cambio, en la percepción de barreras por fatiga/ pereza, obligaciones/ falta de tiempo y ambiente/ instalaciones no se encontraron diferencias estadísticamente significativas $(p>0,05)$.

Intención de ser físicamente activo. Los resultados de la prueba U de Mann-Whitney mostraron cómo en la intención de ser físicamente activo tanto en los varones como en las mujeres no se encontraron diferencias estadísticamente significativas entre adolescentes con normopeso y sobrepeso/ obesidad $(p>0,05)$.

\section{Discusión}

El objetivo de la presente investigación se centraba en comparar la motivación autodeterminada hacia el ejercicio físico, autoconcepto físico, barreras hacia la AF e intención de ser físicamente activo entre adolescentes con y sin sobrepeso/obesidad pertenecientes a la Educación Secundaria Obligatoria. 
Se demuestra que los chicos adolescentes con normopeso tienen una motivación intrínseca asociada a experiencias de diversión y disfrute por la AF, así como mayor regulación integrada e identificada, como formas más autodeterminadas dentro de los tipos de motivación extrínseca, que sus compañeros con sobrepeso u obesidad. Estos últimos en cambio, reportan una mayor regulación externa o tipo de motivación extrínseca menos autodeterminada, asociada a prácticas de $\mathrm{AF}$ para evitar castigos u obtener alguna recompensa. Esto también ocurre en el caso de las chicas con sobrepeso u obesidad respecto a las que tenían normopeso. Por tanto, los resultados coinciden con los del estudio de Hwang y Kim (2013), quienes constataron que los adolescentes obesos y con sobrepeso obtenían mayores niveles de desmotivación y motivación externa hacia laAF que sus compañeros con normopeso. También con el estudio de Power et al. (2011), quienes demostraron una asociación directa entre estar obeso y tener una motivación intrínseca más baja hacia la AF. Además, según dichos autores, aquellos adolescentes intrínsecamente motivados para la AF, tendrían mejor CF y por tanto, menor probabilidad de ser obesos. Riiser, Ommundsen, Smastuen, Londal, Misvaer y Helseth (2014), también advirtieron del papel mediador de la motivación autodeterminada entre la CF y la salud relacionada con la calidad de vida en adolescentes obesos. En este sentido, Olaya-Contreras, Bastidas y Arvidsson (2015) insisten en la idea de que los niños y adolescentes con sobrepeso necesitan una motivación adicional para la adquisición de hábitos saludables y mejoras en la realización de AF asociada a los mismos. Por tanto, resulta necesario incidir en una mejora de su motivación, ya que como demostraron Gourlan et al. (2013), en su intento de explorar las características de la motivación hacia la AF en adolescentes obesos, se muestra que una mayor motivación intrínseca, integrada e identificada se asociaría al cumplimiento de necesidades psicológicas básicas, tales como la autonomía y la necesidad de relacionarse con otros compañeros. Esta última necesidad resultaría de enorme relevancia, en este grupo de población, por las mayores dificultades que encuentran para relacionarse y sentirse integrados en contextos relacionados con la práctica físico-deportiva. En este sentido y de acuerdo a Lindelof, Nielsen y Pedersen (2013), la mayor dificultad se encontraría en el placer limitado que encuentran estos chicos obesos respecto a la realización de actividades activas.

Además, se constata que los chicos adolescentes con normopeso presentan un mejor autoconcepto físico, tanto en la coordinación, grasa corporal, competencia deportiva, autoconcepto físico global y resistencia que sus compañeros con sobrepeso u obesidad. También las chicas con normopeso manifiestan una mejor autopercepción, tanto en la grasa corporal, como en autoconcepto físico global que sus compañeras con sobrepeso u obesidad, mientras que las chicas con sobrepeso u obesidad se perciben como más fuertes que aquellas con normopeso. Por tanto, se confirma, de acuerdo a Reigal-Garrido et al. (2014) en su estudio orientado a examinar las relaciones entre el autoconcepto y diferentes variables de la CF, que la variable con una asociación más fuerte hacia el autoconcepto, sería el porcentaje de grasa corporal. Además, resulta interesante comprobar como aparte del IMC, el estudio de la capacidad aeróbica también resulta un factor clave para determinar el grado de autoconcepto físico de los adolescentes. Prueba de ello es que García-Sánchez et al. (2013), matizaron que aquellos adolescentes con sobrepeso u obesidad que tenían una buena forma física, mostraban un mejor autoconcepto físico que sus compañeros con sobrepeso u obesidad y mala forma física, y similar autoconcepto que aquellos con normopeso, independientemente de su estado de forma. Integrar el conocimiento de los niveles de CF en el análisis realizado, así como la constatación del fenómeno conocido como «fat but fit» abordado en dicho estudio, habría resultado pertinente para una mejor comprensión de la situación en la población objeto de estudio. Por tanto y como señalan Suchert, Hanewinkel e Isensee (2016), la reducción del sedentarismo podría ser un elemento clave en la promoción del autoconcepto físico positivo en los adolescentes.

A partir del presente estudio, también se confirma que, tanto las chicas, como los chicos adolescentes con sobrepeso u obesidad perciben mayores barreras por imagen corporal y ansiedad física y social que sus iguales con normopeso, aunque las evidencias son mayores en el caso de las chicas. Estos datos verifican los hallazgos del estudio de Zabinski et al. (2003), quienes demostraron que aquellos niños con sobrepeso reportaban mayores barreras hacia la AF que los que no lo poseían. Hasta el estudio de dichos autores, se desconocían las posibles diferencias en la percepción de barreras de acuerdo al estado de peso. Al igual que en nuestro estudio, se constataba como principal barrera, sobretodo en las niñas, su propia conciencia del cuerpo como máxima preocupación y obstáculo respecto a la participación en actividades activas. En este sentido, los escolares examinados en nuestro estudio señalaban sentirse incómodos por el aspecto que tenían en ropa deportiva, porque consideraban que su aspecto físico era peor que el de los demás, por percibir que sus compañeros estaban más en forma que ellos, porque pensaban que estos juzgaban su apariencia física y por sentir vergüenza al ser mirados mientras realizaban ejercicio físico. En esta línea, Deforche, De Bourdeaudhuij, Tanghe, Debode, Hills y Bouckaert (2005), advirtieron que esta mayor percepción de inseguridad respecto a la apariencia física en jóvenes con sobrepeso, podía suponer una importante barrera para su participación en actividades deportivas. Nuestros hallazgos también coinciden con el estudio de Deforche et al. (2006), quienes en su intento de medir la actitud hacia la AF mediante la percepción de barreras en adolescentes con normopeso, sobrepeso y obesidad, volvieron a señalar la inseguridad respecto a su apariencia, junto con la percepción de no ser bueno en la práctica, el poco gusto por la misma y problemas físicos, como principales barreras en adolescentes obesos respecto a aquellos con sobrepeso y en estos últimos respecto a los que tenían normopeso. De esta forma y al igual que en el presente estudio, constataban que la percepción de barreras en adolescentes con obesidad y sobrepeso era mayor que en aquellos con normopeso. También con el estudio de Young-Ho (2008), donde se señalaba que aquellos adolescentes con sobrepeso y obesidad eran más propensos a autoimponerse barreras internas, tales como la insatisfacción física y la falta de competencia, directamente asociadas con la ansiedad física y social que supondría exponerse al juicio de los demás. Asimismo, con el estudio de Reesor et al. (2014), donde se confirmaron diferencias significativas en la percepción de barreras de acuerdo al peso, siendo señaladas la conciencia de la apariencia física durante la práctica de AF, la falta de habilidad en la práctica o el ser objeto de burlas durante la participación en la misma como principales impedimentos. En la línea de Fredrickson, Kremer, Swinbum, De Silva y McCabe (2015), consideramos necesario dotar a los escolares con sobrepeso de las herramientas necesarias para que sean capaces de comenzar a concebir su propia imagen corporal de manera satisfactoria, como punto de partida para poder adquirir conductas saludables, como aprender a disfrutar de su participación en actividades físico-deportivas con naturalidad y establecer relaciones sociales con sus pares. En esta misma línea, Zabinski et al. (2003), señalaron que enseñar a los niños a apreciar sus cuerpos y las posibilidades de los mismos, podría ayudarles a reducir sus sentimientos de vergüenza y reparar una autoestima y autoconcepto esenciales para la mejora de su imagen corporal. Para ello, la realización de AF resultaría un medio ideal, tal y como evidenciaron Liu et al. (2015), al comprobar el efecto positivo de un programa de intervención sobre ambas variables. La asunción de barreras interpersonales en una edad clave para su desarrollo psicosocial, podría magnificar la vulnerabilidad de estos jóvenes (Stankov, Olds \& Cargo, 2012), por lo que el establecimiento de programas orientados a modificar conductas para la consecución de estilos de vida saludables y la búsqueda de sostenibilidad para propiciar el mantenimiento de los mismos a largo plazo como gran asignatura pendiente (Smith, Straker, McManus \& Fenner, 2014), se antojan necesarios para estos grupos de población. En este sentido, Deforche, De Bourdeaudhuij, Tanghe, Hills y De Bode (2004), ya demostraron la eficacia de intervenciones para disminuir barreras y potenciar mejoras en los niveles de AF de niños y adolescentes con obesidad.

Respecto a la intención de ser físicamente activo, no se cumple que aquellos adolescentes con sobrepeso u obesidad tengan una menor intención de serlo en el futuro. De acuerdo al estudio de Moreno et al. 
(2007) donde se constataba que de todos los factores del autoconcepto físico, la competencia percibida se concebía como el principal predictor de la intencionalidad de ser físicamente activo y dado que en el presente estudio se ha confirmado que los chicos con normopeso presentaban una mejor percepción de su competencia deportiva, se podían intuir unos resultados diferentes. No obstante, dichos autores también señalaron la percepción de fuerza, CF, autoestima y apariencia física, como elementos predictores de dicha intención. Por tanto, conocer la influencia de dichas variables, así como de algunos factores analizados en el presente estudio (motivación, autoconcepto físico, percepción de barreras) y otros muchos presentes en la literatura actual, como factores predictores de ser o no físicamente activo en el futuro, podría resultar interesante.

La comprensión de los factores predictores o determinantes de la AF analizados, resulta relevante por ser estos potencialmente modificables. Por tanto, el conocimiento facilitado en el presente estudio, podría constituir una buena base teórica sobre la cual construir e implementar programas efectivos para mejorar los niveles de AF de los escolares. De acuerdo a Wilkinson et al. (2016), adaptar estos programas de intervención orientados a promocionar $\mathrm{AF}$ a las evidencias encontradas sobre correlaciones existentes entre dichos determinantes y el nivel de AF de los adolescentes, ayudaría a aumentar los índices de participación activa en escolares, especialmente en aquellos con sobrepeso u obesidad.

\section{Referencias}

Abarca-Sos, A., Murillo, B., Julián, J.A., Zaragoza, J. \& Generelo, E. (2015). La Educación Física: ¿Una oportunidad para la promoción de la actividad física? Retos. Nuevas tendencias en Educación Física, Deporte y Recreación, 28, 155-159.

Ajzen, I. (1985). From Intention to Actions: A Theory of Planned Behavior, en J. Kuhl and J. Beckmann (eds) Action-Control: From Cognition to Behaviour, 11-39. Heidelberg: Springer.

Atkin, A., Van Sluijs, E., Dollman, J., Taylor, W. \& Stanley, R. (2016). Identifying correlates and determinants of physical activity in youth: How can we advance the field? Preventive Medicine, 87, 167-169.

Beltrán, V., Sierra, A.C., Jiménez,A., González-Cutre, D., Martínez, C. \& Cervello, E. (2017). Diferencias según género en el tiempo empleado por adolescentes en actividad sedentaria y actividad física en diferentes segmentos horarios del día. Retos. Nuevas tendencias en Educación Física, Deporte y Recreación, 31, 3-7.

Biddle, S., Gorely, T. \& Stensel, D. (2004). Health-enhancing physical activity and sedentary behaviour in children and adolescents. Journal of Sports Sciences, 22(8), 679-701.

Bush, P.L. \& MacDonald, C. (2015). Promoting Physical Activity Among Adolescents: Recommendations From Correlation Research. Retos. Nuevas tendencias en Educación Física, Deporte y Recreación, 28, 184-189.

Capdevila, L. (2005). Actividad física y Salud. Barcelona: Miracle.

Cole, T. J., Bellizzi, M. C., Flegal, K. M. \& Dietz, W. H. (2000). Establishing a standard definition for child overweight and obesity worldwide: International survey. BMJ, 320(7244), 1240-1243.

Cole, T. J., Flegal, K. M., Nicholls, D. \& Jackson, A. A. (2007). Body mass index cut offs to define thinness in children and adolescents: International survey. BMJ, 335(7612), 194-197.

Deci, E. \& Ryan, R. (1985). Intrinsic motivation and self-determination in human behavior. New York: Plenum.

Deforche, B., De Bourdeaudhuij, I., Tanghe, A., Hills, A. \& De Bode, P. (2004). Changes in physical activity and psychosocial determinants of physical activity in children and adolescents treated for obesity. Patient Education and Counseling, 55, 407-415.

Deforche, B., De Bourdeaudhuij, I., Tanghe, A., Debode, P., Hills, A. \& Bouckaert, J. (2005). Role of physical activity and eating behaviour in weight control after treatment in severely obese children and adolescents. Acta Paediatrica, 94(4), 464-470.

Deforche, B., De Bourdeaudhuij, I. \& Tanghe, A. (2006). Attitude toward physical activity in normal-weight, overweight and obese adolescents. Journal of Adolescent Health, 38, 560-568.

Dolenc, P. \& Pisot, S. (2010). A review of correlates of physical activity in children and adolescents. Didactica Slovenica-Pedagoska Obzorja, 25(2), 85-96.

Downs, D., Savage, J. \& DiNallo, J.(2013). Self-Determined to Exercise? Leisure-Time Exercise Behavior, Exercise Motivation, and Exercise Dependence in Youth. Journal of Physical Activity \& Health, 10(2), 176-184.

Fernández-Bustos, J., González-Martí, I., Contreras, O. \& Cuevas, R. (2015). Relationship between body image and physical self-concept in adolescent females. Revista Latinoamericana de Psicología,47(1), 25-33.

Fredrickson, J., Kremer, P., Swinbum, B., De Silva, A. \& McCabe, M. (2015). Weight perception in overweight adolescents:Associations with body change intentions, diet and physical activity. Journal of Health Psychology, 20(6), 774-784.

García-Sánchez, A., Burgueño-Menjibar, R., López-Blanco, D. \& Ortega, F. (2013). Condición física, adiposidad y autoconcepto en adolescentes. Estudio piloto. Revista de Psicología del Deporte, 22(2), 453-461.

González-Cutre, D., Sicilia, A. \& Fernández, A. (2010). Hacia una mayor comprensión de la motivación en el ejercicio físico: Medición de la regulación integrada en el contexto español. Psicothema, 22(4), 841-847.

Gourlan, M., Trouilloud, D. \& Sarrazin, P. (2013). Motivational characteristics of obese adolescents toward physical activity: Contribution of self-determination theory. European Review of Applied Psychology, 63(4), 209-218.

Graham, D., Wall, M., Larson, N. \& Neumark-Sztainer, D. (2014). Multicontextual Correlates of Adolescent Leisure-Time Physical Activity. American Journal of Preventive Medicine, 46(6), 605616.

Grao-Cruces, A., Fernández-Martínez, A., Nuviala,A. \& Pérez-Turpin, J.A. (2015). Intention to be Physically Active is Influenced by Physical Activity and Fitness, Sedentary Behaviours, and Life Satisfaction inAdolescents. Collegium antropologicum, 39(3), 567573.

Gunnell, K., Brunet, J., Wing, E. \& Belanger, M. (2015). Measuring Perceived Barriers to Physical Activity in Adolescents. Pediatric Exercise Science, 27(2), 252-261.

Hein, V., Muur, M. \& Koka, A. (2004). Intention to be Physically Active after School Graduation and Its Relationship to Three Types of Intrinsic Motivation. European Physical Education Review, 10(1), 5-19.

Hwang, J. \& Kim, Y. (2013). Physical Activity and its Related Motivational Attributes in Adolescents with Different BMI. International journal of behavioral medicine, 20(1), 106-113.

Lindelof, A., Nielsen, C. \& Pedersen, B. (2013). A Qualitative, Longitudinal Study Exploring ObeseAdolescents'Attitudes Toward Physical Activity. Journal of Physical Activity \& Health, 10(1), 113-121.

Liu, M., Wu, L. \& Ming, Q. (2015). How Does Physical Activity Intervention Improve Self-Esteem and Self-Concept in Children and Adolescents? Evidence from a Meta-Analysis. Plos One, 10(8), e0134804.

Marques, A., Peralta, M., Martins, J., Sarmento, H., Routen, A.C. \& Carreiro, F. (2016). Psychosocial correlates of organized physical activity in Portuguese urban youth. Motriz: Revista de Educação Física, 22(4), 327-334.

Marsh, H. W., Richards, G. E., Johnson, S., Roche, L. \& Tremayne, P. (1994). Physical self description questionnaire: psychometric properties and a multitrait-multimethod analysis of relations to existing instruments. Journal of Sport \& Exercise Psychology, 16(3), 270-305.

Marsh, H.W., Martin, A.J. \& Jackson, S. (2010). Introducing a Short Version of the Physical Self Description Questionnaire: New Strategies, Short-Form Evaluative Criteria, and Applications of 
Factor Analyses. Journal of Sport \& Exercise Psychology, 32(4), 438-482.

Mayorga-Vega, D. \& Viciana, J. (2014). Adolescents’ physical activity in physical education, school recess, and extra-curricular sport by motivational profiles. Perceptual \& Motor Skills, 118(3), 663-679.

Moreno, J. A., Moreno, R. \& Cervelló, E. (2007). El autoconcepto físico como predictor de la intención de ser físicamente activo. Psicología y Salud, 17(2), 261-267.

Murtagh, S., Rowe, D., McMinn, D. \& Nelson, N. (2012). Predicting Physical Activity Intentions and Behavior in Children and Adolescents. Research Quarterly for Exercise and Sport, 83, A78A79.

Noriega, M.J., Jaén, P., Santamaría,A.,Amigo, M.T.,Antolín, O., Casuso, I., ... De-Rufino, P.M. (2015). Hábitos sedentarios en adolescentes escolarizados de Cantabria. Retos. Nuevas tendencias en Educación Física, Deporte y Recreación, 27, 3-7.

Niñerola, J., Capdevilla, L. \& Pintanel, M. (2006). Barreras percibidas y actividad física: el autoinforme de barreras para la práctica de ejercicio físico*. Revista de Psicología del Deporte, 15(1), 53-69.

O’Dea, J. (2006). Self-concept, self-esteem and body weight in adolescent females: athree-year longitudinal study. Journal of Health Psychology, 11(4), 599-611.

Olaya-Contreras, P., Bastidas, M. \& Arvidsson, D. (2015). Colombian children with overweight and obesity need additional motivational support at school to perform health-enhancing physical activity. Journal of Physical Activity \& Health, 12(5), 604-609.

Oviedo, G., Sánchez, J., Castro, R., Calvo, M., Sevilla, J.C., Iglesias, A., ... Guerra, M. (2013). Niveles de actividad física en población adolescente: estudio de caso. Retos. Nuevas tendencias en Educación Física, Deportey Recreación, 23, 43-47.

Owen, K., Smith, J., Lubans, D., Ng, J. \& Lonsdale, C. (2014). Selfdetermined motivation and physical activity in children and adolescents: A systematic review and meta-analysis. Preventive Medicine, 67, 270-279.

Park, J. (2011). Predicting adolescents' intention for physical activity and behavior using the Theory of Planned Behavior: the roles of family support and personality. Korean Society of SportPsychology, 22(1), 129-144.

Park, H. \& Kim, N. (2008). Predicting Factors of Physical Activity in Adolescents: A Systematic Review. Asian Nursing Research, 2(2), 113-128.

Power, T., Ullrich-French, S., Steele, M., Daratha, K. \& Bindler, R. (2011). Obesity, cardiovascular fitness, and physically active adolescents' motivations for activity: A self-determination theory approach. Psychology of Sport and Exercise, 12(6), 593-598.

Pulido, J.J., Sánchez-Oliva, D., Sánchez-Miguel, P.A., González-Ponce, I. \& García-Calvo, T. (2016). Proyecto MÓVIL-ÍZATE: Fomento de la actividad física en escolares mediante las Apps móviles. Retos. Nuevas tendencias en Educación Física, Deporte y Recreación, 30, 3-8.

Raudsepp, L., Viira, R. \& Hannus, A. (2010). Prediction of physical intention and behavior in a longitudinal simple of adolescent girls. Perceptual \& Motor Skills, 110(1), 3-18.

Reesor, L., Maeda, H., Raedeke, T., McMillan, A. \& DuBose, K. (2014). Barriers \& Physical Activity Participation Among Overweight/Obese and Healthy Weight Children. Medicine and Science in Sports and Exercise, 46(5), 858-858.

Reigal-Garrido, R., Becerra-Fernández, C., Hernández-Mendo, A. \& Martín-Tamayo, I. (2014). Relationships of self-concept with physical fitness and body composition in a sample of adolescents. Anales de Psicología, 30(3), 1070-1085.

Riiser, K., Ommundsen, Y., Smastuen, M., Londal, K., Misvaer, N. \& Helseth, S. (2014). The relationship between fitness and healthrelated quality of life and the mediating role of self-determined motivation in overweight adolescents. Scandinavian Journal of Public Heatlh, 42(8), 766-772.
Roberts, V., Maddison, R., Magnusson, J. \& Prapavessis, H. (2010). Adolescent Physical Activity: Does Implementation Intention Have a Role? Journal of Physical Activity \& Health, 7(4), 497-507.

Ruiz, J., España-Romero, V., Castro-Piñero, J., Artero, E., Ortega, F., Cuenca-García, M., ... Castillo, M.J. (2011). Batería ALPHAFitness: test de campo para la evaluación de la condición física relacionada con la salud en niños y adolescentes. Nutrición Hospitalaria, 26(6), 1210-1214.

Sallis, J., Prochaska, J. \& Taylor, W. (2000). A review of correlates of physical activity of children and adolescents. Medicine and Science and in Sports and Exercise, 32(5), 963-975.

Smith, K., Straker, L., McManus, A. \& Fenner, A. (2014). Barriers and enablers for participation in healthy lifestyle programs by adolescents who are overweight: a qualitative study of the opinions of adolescents, their parents and community stakeholders. BMC Pediatrics, 14(53), 1-14.

Stankov, I., Olds, T. \& Cargo, M. (2012). Overweight and obese adolescents: what turns them off physical activity?. International Journal of Behavioral Nutrition and Physical Activity, 9(53), 1-15.

Sterdt, E., Liersch, S. \& Walter, U. (2014). Correlates of physical activity of children and adolescents: A systematic review of reviews. Health Education Journal, 73(1), 72-89.

Suchert, V., Hanewinkel, R. \& Isensee, B. (2016). Screen time, weight status and the self-concept of physical attractiveness in adolescents. Journal of Adolescence, 48, 11-17.

Vallerand, R. J. \& Rousseau, F. L. (2001). Intrinsic and extrinsic motivation in sport and exercise: A review using the hierarchical model of intrinsic and extrinsic motivation. En R. N. Singer, H. A. Hausenblas, and C. M. Janelle(Eds.),Handbookof sport psychology (pp. 389-416). New York: Wiley.

Van der Horst, K., Paw, M., Twisk, J. \& Van Mechelen, W. (2007). A brief review on correlates of physical activity and sedentariness in youth. Medicine and Science in Sports and Exercise, 39(8), 12411250.

Velez, A., Golem, D. \& Arent, S. (2010). The impact of a 12-week resistance training program on strength, body composition, and self-concept of Hispanic adolescents. Journal of strength and conditioning research, 24(4), 1065-1073.

Viciana, J., Martínez-Baena,A. \& Mayorga-Vega, D. (2016). Moderateto-vigorous physical activity levels in physical education, school recess and after-school time. Influence of gender, age, and weight status. Journal of Physical Activity \& Health, 13(10), 1117-1123.

Wilkinson, A., Miller, E., Koehly, L., Daniel, C. \& Forman, M. (2016). Correlates of Physical Activity Differ by Sex and Country of Birth Among Mexican-Heritage Youth. Journal of Immigrant and Minority Health, 1-8.

Villa-González, E., Ruiz, J. \& Chillón, P. (2016). Recomendaciones para implementar intervenciones de calidad de promoción del desplazamiento activo al colegio. Retos. Nuevas tendencias en Educación Física, Deporte y Recreación, 30, 159-161.

Wilson, P.M., Rodgers, W.M., Loitz, C.C. \& Scime, G. (2006). «It’s who I am... really!». The importance of integrated regulation in exercise contexts. Journal of Applied Biobehavioral Research, 11(2), 79-104.

Young-Ho, K. (2008). Adolescents' Physical Activity and its related perceived benefits and barriers according to body fat. Korean Society of Sport Psychology, 19(4), 73-86.

Zabinski, M., Saelens, B., Stein, R., Hayden-Wade, H. \& Wilfley, D. (2003). Overweight children's barriers to and support for physical activity. Obesity Research, 11(2), 238-246.

Zook, K., Saksvig, B., Kuo, J. \& Young, D. (2012). Associations between measured and perceived weight status and physical self concept and physical activity among adolescent girls. Annals of Behavioral Medicine, 43, S111-S111. 